\title{
First Report of Sclerotinia White Rot Caused by Sclerotinia nivalis on Panax ginseng in Korea
}

\author{
Hye Sun Cho*, Jeong-Sup Shin, Jae-Hyun Kim, Tae-Kyun Hong, Dae-Hui Cho and Je Yong Kang \\ Ginseng Resource Research Laboratory, Korea Ginseng Corporation, Daejeon 305-805, Korea \\ (Received on September 10, 2012; Revised on January 25, 2013; Accepted on February 5, 2013)
}

\begin{abstract}
Sclerotinia white rot disease was observed on 5 and 6-year-old ginseng (Panax ginseng) roots in Hongchun, Cheorwon, and Yanggu, Gangwon Province, Korea from 2006 to 2010. Symptoms included a brownish watery soft rot of the roots, and black sclerotia were often found on the rotten roots. The causal agent of the disease was identified as Sclerotinia nivalis based on cultural characteristics and sequence analyses of the internal transcribed spacer region of rDNA and $\beta$-tubulin gene with $100 \%$ sequence similarity. Pathogenicity tests were performed on 2-year-old ginseng roots with mycelium plugs without wounds. A watery soft rot of the roots and black sclerotia were observed 10 days after inoculation. These symptoms were identical to those observed on naturally infected roots. The same fungus was re-isolated from the lesions induced by artificial inoculation. This is the first report of sclerotinia white rot caused by $S$. nivalis on P. ginseng in Korea.
\end{abstract}

Keywords : $\beta$-tubulin gene, ITS region of rDNA, Panax ginseng, Sclerotinia nivalis, Sclerotinia white rot

Ginseng (Panax ginseng C. A. Meyer), a perennial medicinal herb in the Araliaceae family, is one of the most economically important crop plants and is usually cultivated in the field for 4-6 years. This results in problems such as root disease by various soil pathogens, including Botrytis, Cylindrocarpon, Pythium, and Sclerotinia. Ginseng white rot caused by the genus Sclerotinia is one of the most important ginseng root diseases in Korea (Park, 1985). Both the incidence and progress of the disease are particularly affected by temperature. The fungus is mesophilic, and the optimum temperature for mycelial growth is $20^{\circ} \mathrm{C}$ (Park, 1985; Saito, 1997) thus, the disease occurs in most ginseng growing regions and is particularly known to be a limiting factor for ginseng cultivation on alpine and sloped-land in Gangwon Province.

A disease described as white rot has been reportedly associated with Sclerotinia panacicola in Korea (Nakata, 1916), although a description of the mycological characteristics and pathogenicity has not been conducted. Park (1985) reported that the fungus causing ginseng white rot is similar to $S$. minor in some cultural and morphological characters. Sclerotial disease caused by S. sclerotiorum on ginseng was reported in 1916 in the United States (Whetzel et al., 1916). Traditional criteria

*Corresponding author

Phone) +82-42-870-3134, Fax) +82-42-870-3117

Email) hscho@kgc.or.kr such as morphological, cultural, and biological characters are usually used to identify the genus Sclerotinia. However, traditional criteria offer considerable difficulties due to similarities in morphological, cultural, and biological characters among Sclerotinia species. Thus, molecular approaches have been used increasingly to support morphological identification of filamentous fungi, and their application has led to a reconsideration of several genera (Bruns et al., 1991). Analysis of ribosomal DNA sequences has become a common tool in modern systematics and has been used to establish phylogenetic relationships among many fungal species (Guadet et al., 1989; Peterson and Kurtzman, 1991). Some proteincoding genes have been used to phylogenetically analyze fungi at the species level. Protein-coding genes have advantages as molecular markers compared to ribosomal genes, as they offer a large number of unlinked sources of phylogenetic information (Geiser et al., 1998; Glass and Donaldson, 1995; Hibbette and Vilgalys, 1991). We collected Sclerotinia isolates from lesions of sclerotinia white rot on ginseng in Gangwon Province for their correct identification.

The objective of this study was to identify the causal agent associated with ginseng white rot disease in Korea based on cultural characteristics, molecular phylogeny, and pathogenicity.

Disease occurrence and symptoms. Sclerotinia white rot disease was found on 5 and 6-year-old ginseng roots in Hongchun, Cheorwon, and Yanggu in Gangwon 

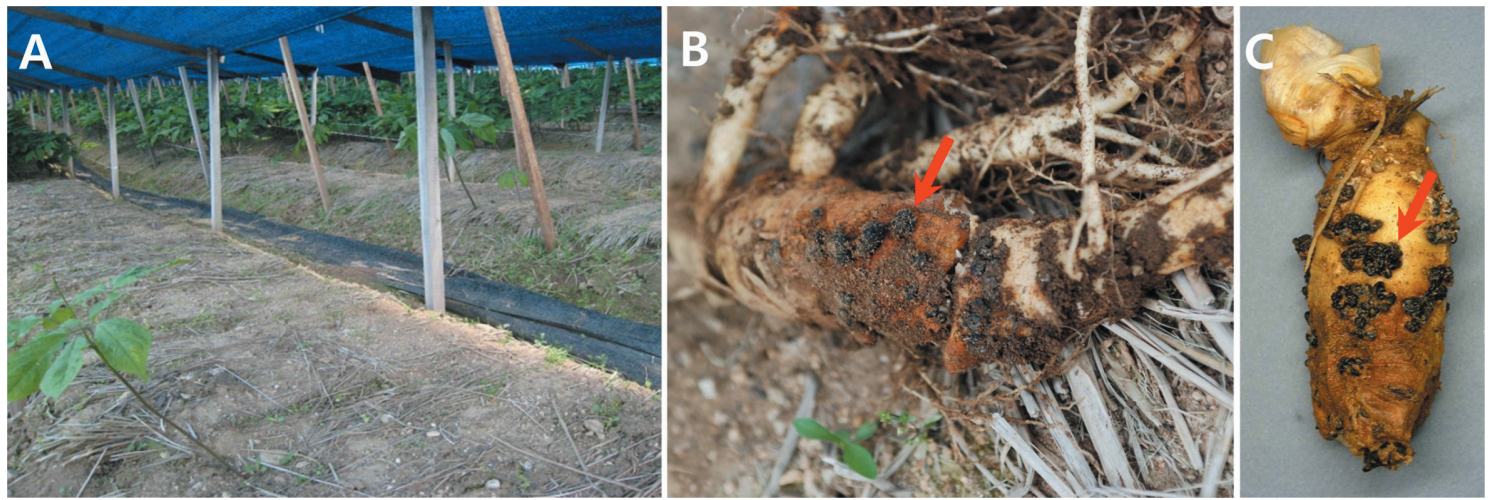

Fig. 1. Symptoms of Sclerotinia white rot on ginseng Yanggu. A: Natural infection in a 6-year-old ginseng field, B, C: infected roots.
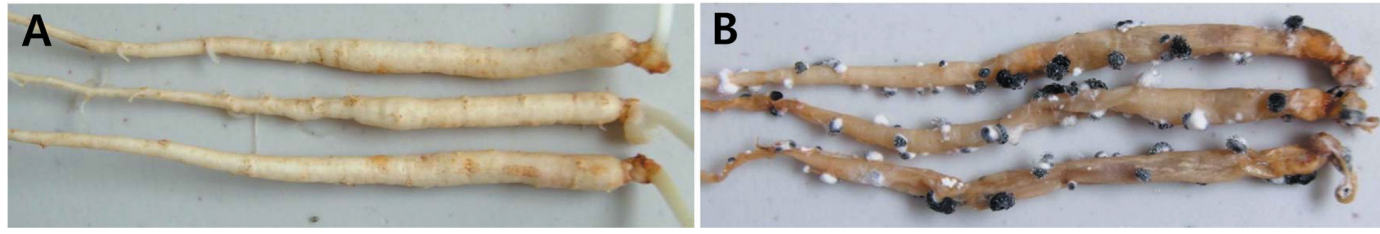

Fig. 2. Pathogenicity of Sclerotinia isolates on 2-year-old ginseng roots at 10 days after inoculation. A: control, B: inoculated.

Province from 2006 to 2010. The ginseng beds with total plant missing were severely infected with sclerotinia white rot (Fig. 1A). Leaf symptoms included wilting foliage that became discolored and desiccated. Infected roots had a brownish watery soft rot and black sclerotia, which varied in shape, and was often produced on rotten roots (Fig. 1B and 1C).

Fungal isolation and pathogenicity. Twenty Sclerotinia isolates were obtained from lesions of white rot on ginseng. The sclerotia from the lesions were sterilized with $70 \%$ ethanol for $30 \mathrm{sec}$ and $1 \% \mathrm{NaOCl}$ for 1 minute, followed by washing in sterile distilled water. The sclerotia were placed on potato dextrose agar (PDA) amended with $0.03 \%$ streptomycin and incubated at $20^{\circ} \mathrm{C}$ for 4 days. Fungal mycelia grown from the sclerotia were transferred to PDA slants.

An inoculum was prepared with mycelial plugs of six representative isolates grown on PDA at $20^{\circ} \mathrm{C}$ for 7 days to determine pathogenicity of the fungus. Twoyear-old ginseng roots were inoculated by placing mycelial plugs without wounds on the root surface and placing the roots on moistened paper towels in plastic boxes. The plastic boxes were kept in a growth chamber at $20^{\circ} \mathrm{C}$ for 10 days. The initial brownish lesion symptoms were observed on the root inoculation sites 5 days after inoculation. A watery soft rot of the roots and black sclerotia were observed 10 days after inoculation (Fig. 2B). These symptoms were identical to those observed on naturally infected roots. No visible symptoms were observed on roots left without the fungal inoculation (Fig. 2A). The same fungus was re-isolated from the lesions induced by artificial inoculation.

Identification of the fungal isolate. To examine cultural characteristics of the 20 isolates, $5 \mathrm{~mm}$ diameter mycelial disks from PDA cultures of the isolates were transferred to PDA in $9 \mathrm{~cm}$ diameter Petri dishes. The Petri dishes were incubated in the dark at $20^{\circ} \mathrm{C}$ for 14 days. Four species of Sclerotinia obtained from the Korea Agricultural Culture Collection (KACC), National Academy of Agricultural Science, Rural Development Administration, Suwon were included to broaden the comparison (Table 1). Colonies of the fungus from ginseng on PDA consisted of snowy white mycelia with globose to irregular and black sclerotia (Fig. 3A). The sclerotia were not seen in the center of the PDA and measured 1.7-2.5 $\times 1.4-2.4 \mathrm{~mm}$. These characteristics were similar to those of the Sclerotinia nivalis isolate (KACC45150) on Aralia elata, which is in the Araliaceae family (Fig. 3B). All isolates from white rot on ginseng have very similar colony characters. The colonies of $S$. minor reference isolates on PDA were white to brown, and small globose sclerotinia were produced on the medium that were $0.3-1.9 \times 1.4-2.4 \mathrm{~mm}$ in size (Fig. 3C). All $S$. sclerotiorum reference isolates formed fewer but larger sclerotia than those of the ginseng isolates near the colony margin. The sclerotia were $2.0-6.7 \times 2.0-$ 
Table 1. List of Sclerotinia isolates used for the molecular phylogenetic analysis

\begin{tabular}{clll}
\hline \hline Species & Isolates No. & \multicolumn{1}{c}{ Host } & \multicolumn{1}{c}{ Location } \\
\hline S. nivalis & KGC-S0601 & Panax ginseng & Hongchun, Gangwon \\
& KGC-S0607 & Panax ginseng & Hongchun, Gangwon \\
& KGC-S0613 & Panax ginseng & Hongchun, Gangwon \\
& KGC-S0707 & Panax ginseng & Cheorwon, Gangwon \\
& KGC-S1001 & Panax ginseng & Yanggu, Gangwon \\
Reference isolates & & & Gapyeong, Gyeonggi \\
S. nivalis & KACC 45150 & Aralia elata & Seogwipo, Jeju \\
S. sclerotiorum & KACC 40172 & Citrus sinensis & - \\
& KACC 40457 & Lactuca sativa & Namyangju, Gyeonggi \\
& KACC 40922 & Pimpinella brachycarpa & Jeju \\
& KACC 41064 & Brassica napus & Hadong, Gyeongnam \\
& KACC 41065 & Capsicum annuum & Jeju \\
KACC 41069 & Solanum tuberosum & Masan, Gyeongnam \\
KACC 42223 & Chrysanthemum & Incheon, Gyeonggi \\
& KACC 45153 & Brassica oleracea var. acephala & Namyangju, Gyeonggi \\
& KACC 41066 & Brassica campestris ssp. pekinensis & Namyangju, Gyeonggi
\end{tabular}

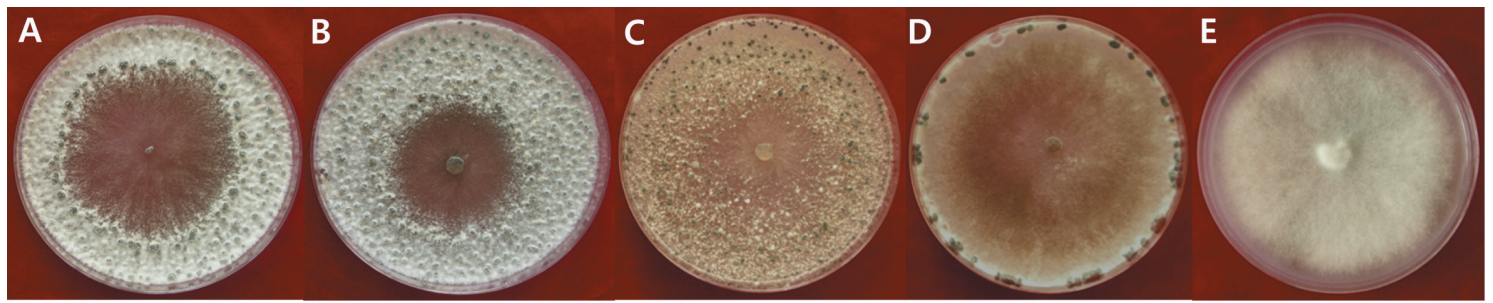

Fig. 3. Colony characters of Sclerotinia species cultured on potato dextrose agar (PDA) at $20^{\circ} \mathrm{C}$ for 14 days in the dark. A: S. nivalis (KGC-S0613), B: S. nivalis (KACC45150), C: S. minor (KACC41066), D: S. sclerotiorum (KACC41069), E: S. trifoliorum (KACC41270).

$4.5 \mathrm{~mm}$ in size (Fig. 3D). The S. trifolium isolate did not produce sclerotia on PDA (Fig. 3E).

Five representative isolates from ginseng and 14 reference Sclerotinia species isolates obtained from the KACC were grown in liquid shake culture in potato dextrose broth for 5 days at $25^{\circ} \mathrm{C}$ for the molecular phylogenetic analysis. Mycelia were collected from the cultures by filtration and transferred to sterile plastic tubes. These samples were frozen at $-70^{\circ} \mathrm{C}$. The frozen mycelia were ground in $1.5 \mathrm{ml}$ tubes. Genomic DNA was isolated using the NucleoSpin ${ }^{\circledR}$ Plant II kit (Macherey-Nagel, Düren Germany), according to the manufacturer's instructions. The rDNA region, including internal transcribed spacer region (ITS) 1, 2, and the $5.8 \mathrm{~S}$ ribosomal gene, were amplified with ITS5
(GGAAGTAAAAGTCGTAACAAGG) and ITS4 (TCC TCCGCTTATTGATATGC) primers (White et al., 1990). The $\beta$-tubulin gene was amplified with the primer sets Bt2a (GGTAACCAAATC GGTGCTGCTTTC) and Bt2b (ACCCTCAGTGTAGTGACCCTTGGC) (Glass and Donaldson, 1995). Polymerase chain reaction (PCR) amplifications were performed using previously reported methods (Glass and Donaldson, 1995; White et al., 1990).

The PCR product was purified by using a Wizard PCR Prep kit (Promega, Madison, WI, USA) and directly sequenced using a BigDye Terminator Cycle Sequencing kit (Applied Biosystems, Forster City, CA, USA), following the manufacturer's instructions. The same primer sets as those used for PCR amplification were 

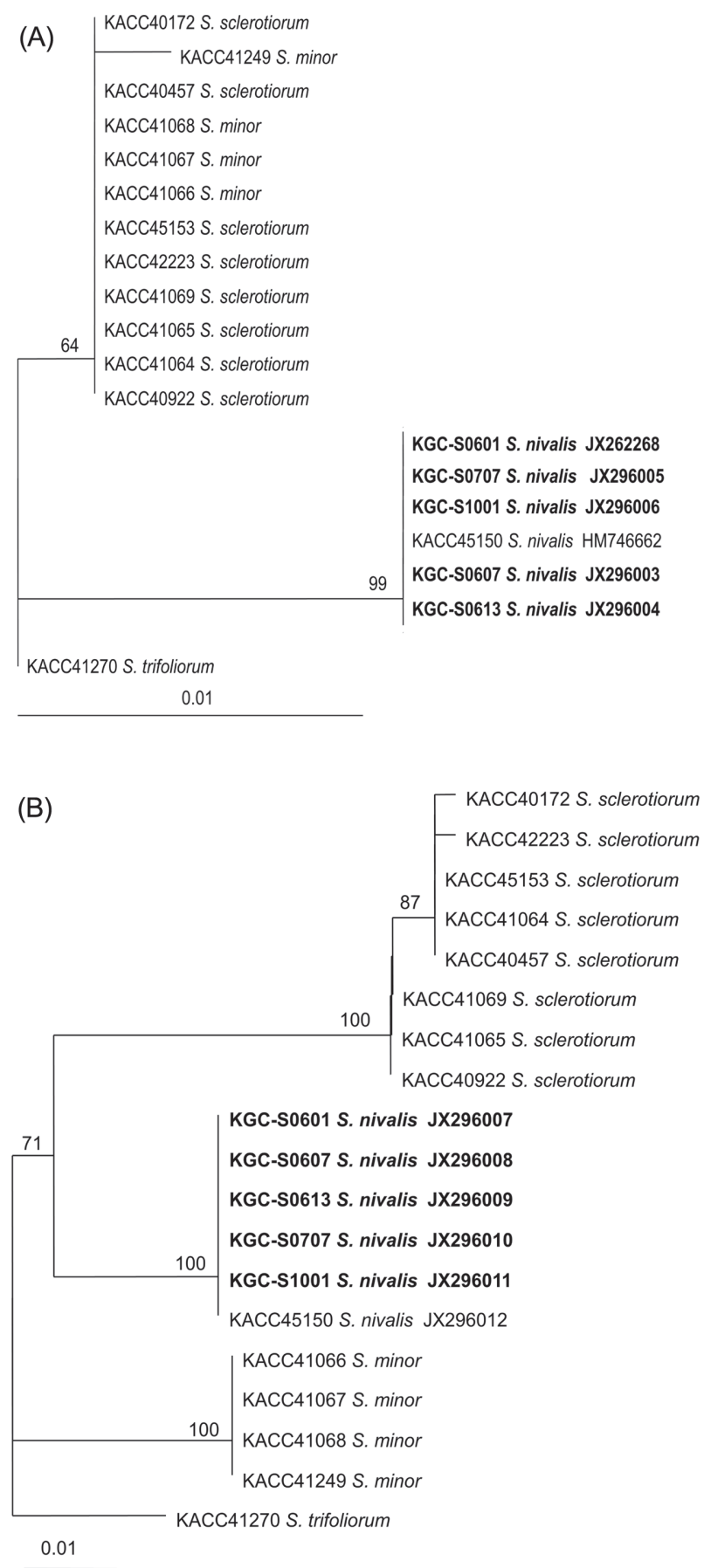

Fig. 4. Neighbor-joining tree based on internal transcribed sequence (ITS) rDNA (A) and $\beta$-tubulin (B) sequences showing the relationships among $S$. nivalis from ginseng and other Sclerotinia species. The values above each branch indicate the percentage levels of bootstrap support $(>50 \%)$ for the branch point based on 1000 resamplings. Bar represents 0.01 substitutions per nucleotide position.

used to sequence both DNA strands. Gel electrophoresis and data collection were performed on an ABI Prism
310 Genetic Analyzer (Applied Biosystems).

Sequences generated from the materials in this study were initially aligned using the CLUSTAL program (Thompson et al., 1997), and then alignment was refined manually using the PHYDIT program version 3.0 (Chun, 1995). Phylogenetic trees were constructed using distance methods. The neighbor-joining method was performed using the Kimura's two-parameter model for the distance analysis. Amplification of the ITS region yielded products of 454-bp. The sequence alignment revealed that all isolates from ginseng were $100 \%$ identical to those of the reference isolate ( $S$. nivalis KACC 45150) isolated from $A$. elata and supported by a $99 \%$ bootstrap value (Fig. 4A). This result was consistent with those of the cultural characters. The $\beta$-tubulin gene sequences from Sclerotinia species was 452-bp. A distance analysis with Kimura's two-parameter model based on the $\beta$-tubulin gene sequence showed that the isolates from ginseng and $S$. nivalis reference isolate (KACC 45150) formed one group with $100 \%$ similarity. This group was clearly distinct from the $S$. minor and $S$. sclerotiorum group (Fig. 4B). This result showed good agreement with the ITS region sequencing data. The resulting sequences of the five isolates from ginseng were deposited in GenBank. The present phylogenetic analysis along with the cultural characteristics indicated that the causal fungus was S. nivalis. Several species of Sclerotinia are commonly found on soil and cause a range of economically important diseases on a variety of crops worldwide (Boland and Hall, 1994; Chang and Kim, 2003; Kim and Cho, 2002; Kim et al., 1999; Melzer et al., 1997; Purdy, 1979). Among them, S. nivalis was first described by Saito (1997) from herbaceous dicots based on sclerotial morphology in culture, the molecular mass of major sclerotia proteins, and the patterns of esterase isozymes in sclerotial extracts. He (1997) reported that the fungus is a mesophile with an optimum temperature for mycelial growth of around $20^{\circ} \mathrm{C}$ and that this fungus attacks Arctium lappa, Chrysanthemum morifolium, Ambrosia elatior, Daucus carota, Angelica acutiloba, Ajuga repans, and Plantago lanceolata. Li et al. (2000) described lettuce drop caused by S. nivalis in central China based on morphological and cultural characteristics, carpogenic germination, and soluble sclerotial proteins. In Korea, Lee et al. (2010) reported that Aralia elata (Miq.) Seem (Japanese angelica tree) is a host for $S$. nivalis, based on cultural characteristics, pathogenicity, and sequence analysis of the ITS regions. However, this is the first report on Sclerotinia white rot caused by $S$. nivalis on $P$. ginseng in Korea.

Global warming and accelerated climate change have recently come to the forefront, which has caused a 
northward shift in the regions suitable for ginseng cultivation in Korea. S. nivalis is mesophilic and found in mountainous areas where temperatures are low, ranging from -5 to $10^{\circ} \mathrm{C}$, and sclerotium formation is observed at 15 and $20^{\circ} \mathrm{C}$ ( $\mathrm{Li}$ et al., 2000). Adams and Ayers (1979) reported that sclerotia of Sclerotinia species generally survive in soil for 3-8 yr. In recent years, ginseng cultivation areas have expanded to mountainous areas of Gangwon Province, so Sclerotinia white rot caused by $S$. nivalis may be a potential threat to ginseng cultivated for 4-6 years in the field. Therefore, detailed epidemiological studies are needed to develop effective control methods for this disease caused by $S$. nivalis.

\section{Acknowledgement}

This study was supported by Research and Development Projects from the Korea Ginseng Corporation.

\section{References}

Abams, P. B. and Ayers, W. A. 1979. Ecology of Sclerotinia species. Phytopathology 69: 896-899.

Abawi, G. S. and Grogan, R.G. 1979. Epidemiology of disease caused by Sclerotinia species. Phytopathology 69: 899-904.

Agrios, G. N. 2005. Plant Pathology, Fifth Edition. Elsevier Academic Press, San Diego, CA. pp. 546-550.

Andrew, M. and Kohn, L. M. 2009. Single nucleotide polymorphismbased diagnostic system for crop-associated Sclerotinia species. Appl. Environ. Microbiol. 17: 5600-5606.

Boland, G. J. and Hall, R. 1994. Index of plant hosts of Sclerotinia sclerotiorum. Can. J. Plant Pathol. 16: 93-108.

Bruns, T. D., White, T. J. and Taylor, J. W. 1991. Fungal molecular systematics. Annu. Rev. Ecol. Syst. 22: 525-564.

Chang, S. W. and Kim, S. K. 2003. First report of Sclerotinia rot caused by Sclerotinia sclerotiorum on some vegetable crops in Korea. Plant Pathology J. 19: 79-84.

Chun, J. S. 1995. Computer-assisted classification and identification of actinomycetes. Ph. D. Thesis, University of Newcastle, Newcastle upon Tyne, UK.

Freeman, J., Ward, E., Calderon, C. and McCartney, H. A. 2002. A polymerase chain reaction (PCR) assay for the detection of inoculums of Sclerotinia sclerotiorum. Eur. J. Plant Pathol. 108: 877-886.

Geiser, D. M., Frisvad, J. C. and Taylor, J. W. 1998. Evolutionary relationships in Aspergillus section Fumigati inferred from partial $\beta$-tubulin and hydrophobin DNA sequences. Mycologia 90: 831-845.

Glass, N. L. and Donaldson, G. C. 1995. Development of primer set designed for use with the PCR to amplify conserved genes from filamentous Ascomycetes. Appl. Environ. Microbiol. 61: $1323-1330$

Guadet, J., Julien, J., Lafay, J. F. and Brygoo, Y. 1989. Phylogeny of some Fusarium species determined by large-subunit rRNA sequence comparison. Mol. Biol. Envol. 6: 227-242.

Hibbette, D. D. and Vilgalys, R. 1991. Evolutionary relationships of Lentinus to the Polyporacear: evidence from restriction analysis of enzymatically amplified ribosomal DNA. Mycology 83: 425-439.

Hirschhäuser, S. and Fröhlich, J. 2007. Multiplex PCR for species discrimination of Sclerotiniaceae by novel laccase introns. Int. J. Food Microbial. 118: 151-157.

Hoegger, P. J., Kilaru, S., James, T. Y., Thacker, J. R. and Kües, U. 2006. Phylogenetic comparison and classification of laccase and related multi-copper oxidase protein sequences. FEBS J. 273: 2308-2326.

Holst-Jensen, A., Vaage, M. and Schumacher, T. 1998. An approximation to the phylogeny of Sclerotinia and related genera. Nord. J. Bot. 18: 705-719.

Kim, W. G., Cho, W. D. and Jee, H. J. 1999. Occurrence of Sclerotinia rots on cucurbitaceous vegetable crops in greenhouses. Korean J. Mycol. 27: 198-205. (In Korean)

Kim, W. G. and Cho, W. D. 2002. Occurrence of Sclerotinia rots on composite vegetable crops and causal Sclerotinia spp. Mycobiology 30: 41-46.

Kohn, L. M., Petche, D. M., Bailey, S. R., Novak, L. A. and Anderson, J. B. 1988. Restriction fragment length polymorphism in nuclear and mitochondrial DNA of Sclerotinia species. Phytopathology 79: 1047-1051.

Lee, C. K., Lee, S. H., Choi, Y. J., Park, M. J. and Shin, H. D. 2010. Sclerotinia rot of Aralia elata caused by Sclerotinia nivalis in Korea. Plant Pathology J. 26: 426.

Li, G. Q., Wang, D. B., Jiang, D. H., Huang, H. C. and Laroche, A. 2000. First report of Sclerotinia nivalis on lettuce in central China. Mycol. Res. 104: 232-237.

Lumsden, R. D. 1979. Histology and physiology of pathogenesis in plant disease caused by Sclerotinia species. Phytopathology 69: 890-896.

Melzer, M. S., Smith, E. A. and Boland, G. J. 1997. Index of plant hosts of Sclerotinia minor. Can. J. Plant Pathol. 19: 272-280.

Nakata, K. 1916. Studies on ginseng diseases. Chosun (Korea) Model Exper. Station Report (in Japanese) 2: 163.

Park, K. J. 1985. Sclerotinia species causing ginseng white rot in Korea. MS thesis. Seoul National University, Seoul, Korea.

Peterson, S. W. and Kurtzman, C. P. 1991. Ribosomal RNA sequence divergence among sibling yeast species Syst. Appl. Microbiol. 14: 124-129.

Price, K. and Calhoun, J. 1975. Pathogenicity of isolates of Sclerotinia sclerotiorum (Lib.) de Bary to several hosts. Phytopathol. Z. 83: 232-238.

Purdy, L. H. Sclerotinia sclerotiorum: History, disease and symptomatology, host range, geographic distribution and 
impact. Phytopathology 69: 875-880.

Putnam, M. L. 2004. First report of stem rot of rosemary caused by Sclerotinia sclerotiorum in the United States. Plant Pathol. 53: 252.

Saito, I. 1997. Sclerotinia nivalis, sp. nov., the pathogen of snow mold of herbaceous dicots in northern Japan. Mycoscience 38: 227-236.

Thompson, J. D., Gibson, T. J., Plewniak, F., Jeanmougin, F. and Higgins, D. G. 1997. The Clustal windows interface: flexible strategies for multiple sequence alignment aided by quality analysis tools. Nucleic Acids Res. 24: 4876-4882.

Tourneau, D. L. 1979. Morphology, cytology and physiology of
Sclerotinia species in culture. Phytopathology 69: 887-890.

White, T. J., Bruns, T., Lee, S. and Taylor, J. 1990. Amplification and direct sequencing of fungal ribosomal RNA genes for phylogenetics. In: PCR protocols. A guide to methods and applications, ed. by Innis, M. A., Gelfand, D. H., Sninsky, J. J., and White, T. J. pp. 315-322. Academic Press, San Diego.

Whetzel, H. H., Rosenbaum, J., Brann, J. W. and McClintock, J. A. 1916. Ginseng disease and their control. U. S. Dept. Agr. Farmer's Bull. 736: 10-12.

Yu, Y. H. and Ohh, S. H. 1993. Research on ginseng disease in Korea. Korean J. Ginseng Sci. 17: 61-68. (In Korean) 